\title{
Regulación del sistema educativo y desigualdades de aprendizaje en el Uruguay
}

\author{
Juan A. Bogliaccini y Federico Rodríguez
}

RESUMEN

En este artículo se muestra cómo determinados aspectos del sistema uruguayo de educación secundaria pública inciden en rendimientos desiguales de los alumnos. Al utilizar la edición 2006 del Programa Internacional de Evaluación de Estudiantes (PISA) (OCDE, 2006a) resaltan tres aspectos clave de las instituciones reguladoras de la educación secundaria que contribuyen a reproducir las desigualdades iniciales, inhibiendo el papel igualador que orienta al sistema educativo. En primer lugar, el mecanismo de asignación de docentes produce un doble efecto de alta rotación de profesores jóvenes en establecimientos de enseñanza de contextos socioculturales desfavorables, así como un anquilosamiento de aquellos docentes más experimentados en establecimientos de contextos favorables. En segundo lugar, el sistema de distribución de alumnos basado en el radio escolar reproduce tel proceso de segregación residencial existente. Finalmente, con el sistema centralizado de provisión de materiales educativos y tecnológicos no se logra cubrir las necesidades de los establecimientos.

PALABRAS CLAVE

CLASIFICACIÓN JEL

AUTORES
Educación, enseñanza secundaria, rendimiento escolar, calidad de la educación, evaluación, personal docente, establecimientos de enseñanza, material didáctico, Uruguay

I24, I28, D31

Juan A. Bogliaccini es profesor asistente del Departamento de Ciencias Sociales y Políticas de la Universidad Católica del Uruguay. juan.bogliaccini@ucu.edu.uy

Federico Rodríguez es director de la Unidad de Estudios, Investigación e Indicadores del Instituto Nacional de Evaluación Educativa del Uruguay. federico.rodriguez@correo.ucu.edu.uy 


\section{I}

\section{Introducción}

El Uruguay es uno de los países de mayor desarrollo humano de América Latina y el de menor desigualdad en la región ${ }^{1}$. Sin embargo, en la segunda mitad del siglo XX el país experimentó un proceso de estancamiento económico que provocó un lento, pero sostenido empobrecimiento de su matriz sociocultural y un aumento en los niveles de pobreza y desigualdad. El Estado de bienestar fue perdiendo la capacidad de responder a la nueva estructura de riesgos sociales (Filgueira y otros, 2005). Así, la educación secundaria es uno de los sectores de política pública que ha mostrado menor capacidad de dar respuesta a la nueva estructura de riesgos, en parte debido al bloqueo político que el sector ha experimentado en las últimas tres décadas. En efecto, la representación de diversos intereses corporativos en los órganos de dirección, combinada con una crisis de identidad con respecto al propósito social que la educación secundaria debe cumplir, marcan los principales puntos de conflicto.

En este artículo se sostiene que el análisis de las causas de las desigualdades de aprendizaje debe abordarse desde una lógica multinivel. Ni el nivel individual (hogar), ni el nivel del sistema educativo pueden dar cuenta por sí mismos de este problema. El nivel individual ha sido el más examinado en el Uruguay desde el estudio pionero de Rama (1993), siendo los estudios relativos al efecto del hogar de procedencia del alumno en sus logros educativos los más prolíferos en la literatura nacional. El propósito de este trabajo es ayudar a comprender las raíces institucionales de las desigualdades de aprendizaje.

\footnotetext{
${ }^{1}$ Con un coeficiente de Gini de 39,5, el Uruguay es el país de la región con menor nivel de desigualdad. Para una clasificación completa de los niveles de desigualdad globales, véase Banco Mundial, 2014.
}

El argumento presentado es que en el sistema educativo público, que educa al $83 \%$ de los estudiantes de secundaria, no se han conseguido modificar los principales aspectos organizativos en concordancia con el cambio en la estructura de riesgos del país, lo que ha contribuido a la consolidación de las desigualdades de los logros en educación a través de dos vías: la exclusión de alumnos del sistema y la segmentación de calidad entre establecimientos de enseñanza. En términos de dicha segmentación, se analizan tres causas principales: la estabilidad de los docentes en los establecimientos; el tipo de vínculo entre estos planteles y la administración central del sistema; y el mecanismo de asignación de alumnos a los establecimientos educativos.

El desajuste entre los nuevos riesgos sociales y las instituciones que regulan el sistema educacional se ha traducido en un estancamiento de buena parte de los indicadores educativos. Esto es más problemático en la educación secundaria, donde la evidencia indica que el país no ha logrado mejorar ni los niveles de culminación del ciclo básico para los jóvenes de entre 15 y 20 años de edad, ni los niveles de aprobación del segundo ciclo de educación secundaria (véase el gráfico 1).

Este trabajo se organiza de la siguiente manera. Luego de la Introducción, en la sección II del artículo se explican los tres mecanismos de regulación del sistema educacional uruguayo que impiden evitar que las desigualdades socioeconómicas de origen de los alumnos se transformen en desigualdades educativas. A continuación, en la sección III, se presenta el modelo elaborado para el análisis de los datos y se procede a la descripción de las variables incluidas en él. Finalmente, en la cuarta sección se presentan los resultados y en la sección V se discuten las conclusiones. 


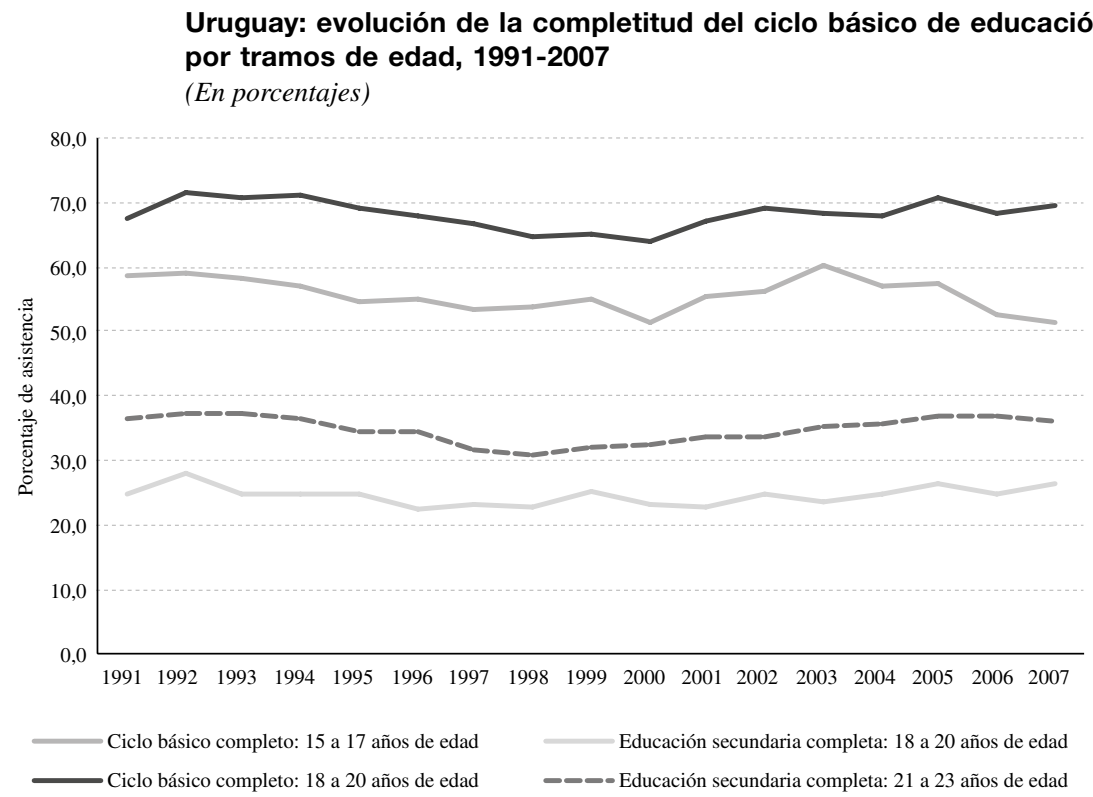

Fuente: elaboración propia sobre la base de datos del Consejo Directivo Central (CODICEN) de la Administración Nacional de Educación Pública (ANEP).

a $1^{\circ}$ a $3^{\text {er }}$ año de educación secundaria.

\section{II}

\section{Regulación y desigualdad: hipótesis sobre vínculos causales}

\section{La desigualdad y la educación: una relación conflictiva}

¿Cuál es el papel de la educación en la búsqueda de la equidad social? Del viejo paradigma se ha heredado la idea funcionalista sobre la educación como factor de integración social y principal agente socializador. Es a partir del Informe Coleman (Coleman, 1966) que comienza a ganar terreno la idea de que las escuelas poco pueden hacer para contribuir a la equidad social. Surgen entonces otros niveles de análisis de los logros en educación, como son la familia, el barrio y el propio establecimiento educacional. En relación con el papel específico que el plantel escolar y el propio sistema educativo tienen en los aprendizajes de los estudiantes, Hanushek y Luque (2001) muestran el desproporcionado papel que desempeña, en países con pocos ingresos, el establecimiento escolar en comparación con la familia en el logro educativo de los estudiantes. Con respecto a América Latina, Cueto (2004) muestra cómo la escuela tiene una mayor incidencia en dicho logro de la que se sostiene en el Informe Coleman.

La Conferencia Mundial sobre la Educación para Todos (Jomtien, Tailandia, 1990) y el Foro Mundial sobre la Educación (Dakar, Senegal, 2000) pusieron como metas globales avanzar en tres dimensiones clave: cobertura, calidad y equidad en la educación. A partir de entonces, se han multiplicado los esfuerzos por medir los resultados y procesos de aprendizaje, de modo de poder evaluar con mayor certeza las políticas a seguir y el papel de los diferentes factores antes expuestos en la determinación de los niveles de logro educativo.

Las corrientes orientadas al estudio de las desigualdades sociales focalizaron su interés en la educación como un medio para minimizarlas. Estas corrientes - como la teoría feminista (Abbott, Tyler y 
Wallace, 1990; Stromquist, 1990)—, el enfoque de las escuelas eficaces (Edmonds, 1986; Fernández, 1999; Hanushek, Link y Woessmann, 2012), la composición social de la escuela y los efectos de los pares (Wilkinson y otros, 2002; Sacerdote, 2001; Graham, 2008; De Giorgi, Pellizzari y Redaelli, 2010) fueron surgiendo e incorporando miradas específicas que permitieran comprender las desigualdades que operaban en la sociedad y cómo el ámbito escolar se hacía eco de ellas o, por el contrario, constituía una forma de combatirlas. En particular, resultan relevantes para la presente investigación los hallazgos reportados por Hanushek y Woessmann (2010) sobre la relación entre las pruebas de logro educativo y el tipo y la calidad de trabajos obtenidos en el mercado laboral. Estos autores denotan cómo esta relación es robusta entre países y regiones, argumento que refuerza la relevancia en materia de política de la pregunta que orienta el presente estudio.

En otros trabajos se enfatiza la importancia de la igualdad de oportunidades (Roemer, 2005; Perera, Llambí y Messina, 2009; Méndez y Zerpa, 2009). En este enfoque, que ilumina más directamente la pregunta sobre la relación entre las regulaciones del sistema educativo y las desigualdades de aprendizaje, se establece que lo importante es otorgar a los individuos las mismas "oportunidades de uso" de sus capacidades. En consecuencia, para minimizar las diferencias en el punto de partida, el sistema educativo debe actuar como igualador de oportunidades procurando que individuos con similar talento y esfuerzo logren el mismo nivel de resultados, independientemente de su entorno familiar o social. Son entonces los factores institucionales (condiciones de aprendizaje) los que permiten lograr mayor equidad en el punto de llegada (DiMaggio, 1982; Vélez, Schiefelbein y Valenzuela, 1994).

\section{El caso del Uruguay: regulación y desigualdad}

El sistema educativo uruguayo se gestiona en forma centralizada y es regulado por el Estado, fundamentalmente por las vías del sistema de inspecciones y del currículo único ${ }^{2}$. En el período 1995-1999, durante la presidencia de Sanguinetti, se produce el proceso más profundo de reforma al sistema educativo en los últimos 70 años, y sus principales estrategias se dirigieron al eje de la equidad

2 El principal canal de comunicación formal del establecimiento educativo con el nivel central del sistema opera a través de la figura del inspector. Este cargo, al que se accede por concurso, tiene la función de evaluar a docentes y directores, así como velar por el correcto funcionamiento de los planteles de enseñanza. en recursos (con énfasis compensatorio) y resultados con modelos centralizados en que se combinaron focalización y universalidad en la asignación de recursos.

Desde el inicio de esta reforma y hasta la presidencia de Vázquez (2005-2009), el sistema educativo enfrentó un conjunto de tensiones estructurales entre el modelo preexistente y el emergente sin poder resolverlas. El debate estuvo rodeado de disputas políticas e ideológicas, generando continuos conflictos entre las autoridades del ámbito educacional y los sindicatos docentes, sobre todo en la educación secundaria. Durante el gobierno de Vázquez, varios de los cambios introducidos por la reforma fueron revertidos, otros fueron desacelerados o estancados, y las principales innovaciones educativas fueron gestionadas desde el exterior del propio sistema, como el Plan CEIBAL, que entrega a cada alumno en la educación pública un computador personal (Martínez, Alonso y Díaz, 2009).

Existen tres aspectos de la regulación actual del sistema educativo que son importantes para comprender la incapacidad que este ha tenido tanto para mantener altos niveles de cobertura, como para evitar que las desigualdades socioeconómicas de origen de los alumnos se transformen en desigualdades de aprendizaje. En primer lugar, el mecanismo de asignación de docentes a los establecimientos educativos produce un doble efecto de alta rotación de profesores jóvenes e inexperimentados en los establecimientos de contexto sociocultural desfavorable y muy desfavorable, y una concentración de maestros con mayor experiencia en los de contexto favorable. En segundo lugar, el sistema de distribución de alumnos en los planteles de enseñanza basado en el radio escolar reproduce el proceso de segregación social existente. Por último, mediante el sistema centralizado de provisión de materiales educativos y tecnológicos no se logran cubrir las necesidades de los establecimientos educacionales, generando una lógica paralela de provisión de materiales basada en la capacidad de los padres de los alumnos para solventar dichas necesidades.

\section{a) Distribución docente por establecimiento educativo}

El mecanismo de asignación de cargos docentes y de dirección en el sistema público contrasta marcadamente con su lógica centralizada. Los estatutos de elección de establecimiento educativo fijan un mecanismo de autonomía a nivel docente para elegir aquel de su preferencia, así como para cambiar de plantel a discreción, dentro del marco de un ordenamiento del cuerpo docente que da prioridad a aquellos profesores con mayor antigüedad en el sistema, el que funciona 
de la siguiente manera: cada año se ponen a elección los cargos que están disponibles. Una vez conocido el total de estos cargos, en cada departamento del país se hace la elección de horas. Si un docente lo desea, puede inscribirse hasta en dos departamentos para dicha elección. Las normas vigentes establecen un complejo mecanismo regido por lo que se conoce como un "orden de precedencia por grado en orden decreciente"3. Básicamente, con este mecanismo se establece que los docentes efectivos eligen antes que los interinos y suplentes; los de mayor grado eligen antes que los de menor grado y, dentro de cada grado, los que tienen mayor puntaje eligen antes que quienes tienen menor puntaje. Los docentes eligen establecimiento educativo por orden de precedencia, pero posteriormente se produce un mecanismo complementario de renuncia al cargo elegido y selección en forma interina de un cargo mejor, que a su vez fue dejado vacante por otro docente. Estas vacantes se producen en forma de goteo por licencias o por profesores que van a desempeñar funciones en la Administración Nacional de Educación Pública (ANEP) o pasan a comisión en otro organismo público.

De este modo, la distribución final de docentes en los establecimientos educativos depende de lo que cada uno de ellos haya decidido al tener que optar en estas condiciones. Ni las autoridades, ni los directores, ni los padres tienen jurisdicción en esta materia ${ }^{4}$. El único límite que existe con respecto a las posibilidades de elección de un docente son las elecciones de los otros profesores o, más precisamente, las elecciones de aquellos mejor ubicados en el "orden de precedencia".

Este sistema privilegia al docente por sobre el estudiante, dado que hace imposible incorporar al sistema algún criterio o incentivo estratégico en la distribución de docentes en los establecimientos educativos. Las consecuencias más notables de esta regulación son la segmentación de profesores según experiencia y la generación de inestabilidad crónica en los cargos de docencia de los planteles de enseñanza de contextos más desfavorables. Esto a su vez genera inequidades a través de dos vías. En primer lugar, debido a la distribución de docentes de mayor experiencia con sesgo hacia los establecimientos educacionales que cumplen con la doble característica de estar ubicados en barrios de contextos más favorables y tener ambos ciclos de educación secundaria

\footnotetext{
3 Véase el Estatuto del Funcionario Docente, particularmente en sus artículos 13 y 14. Este mismo mecanismo se recoge en los reglamentos de los Consejos Desconcentrados.

4 Las autoridades de la ANEP pueden por vía indirecta suprimir, trasladar o crear cargos.
}

$\left(1^{\circ}\right.$ a $3^{\circ}$ año y $4^{\circ}$ a $6^{\circ}$ año, respectivamente). En segundo lugar, a causa de la alta rotación en los establecimientos de contextos más desfavorables, lo que disminuye su capacidad de coordinación, en especial la posibilidad de hacer seguimiento a los alumnos que presentan mayores problemas de aprendizaje (Reimers, 2000; García Huidobro, 2003; Bogliaccini, 2003 y 2007). Ello redunda en mayores tasas de repetición y abandono escolar (ANEP, 1999 y 2002; Filgueira, Fuentes y Rodríguez, 2006). El problema se agudiza en la educación secundaria y va a contramano de lo que la evidencia internacional relaciona con los buenos resultados, puesto que un docente elige cargos en distintos establecimientos educativos, de modo que los últimos en elegir deben dictar clases en otros planteles geográficamente alejados, lo que dificulta tanto la calidad de la enseñanza como la posibilidad de coordinación, aumentando el ausentismo docente (ANEP, 2005 y 2008).

\section{b) Asignación de alumnos a establecimientos educativos por radio escolar}

La asignación de alumnos al plantel educativo por radio escolar, es decir, según un criterio de proximidad geográfica, en una sociedad con niveles de desigualdad relativamente altos y, aún más importante, con niveles de segregación socio-residencial también elevados, produce un problema de segmentación escolar (Kaztman, 1999 y 2001; Filgueira y Bogliaccini, 2004; Kaztman y Retamoso, 2006). En sociedades con marcados niveles de desigualdad existen dos grandes fuentes generadoras de riesgos de segregación educativa: la base territorial de reclutamiento y la existencia de una oferta segmentada en materia de calidad y costos. Por segregación educativa debe entenderse aquella situación en la que la experiencia en educación del individuo, o de un grupo de individuos dentro de la sociedad, tiende a desarrollarse y compartirse esencialmente con individuos de su misma extracción social. Esta segregación va en detrimento no solo de la formación del carácter ciudadano de la población, sino también del aprendizaje y logro educativo (Kaztman, 2001; Kaztman y Filgueira, 2001; ANEP, 2002).

Tal proceso de distribución de alumnos por radio escolar afecta a la equidad en los aprendizajes, porque debilita el "efecto pares" en el interior del sistema (García Huidobro, 2000; Reimers, 2000; ANEP, 2002). Sin embargo, dada la cantidad de planteles de educación secundaria y su distribución geográfica, la segmentación en este ciclo se manifiesta en forma diferente, incluso contra-intuitiva, con respecto al proceso de segmentación en el ciclo de educación primaria (que comprende 6 años). En este último, la segmentación se produce 
por la existencia de establecimientos cuyo alumnado es homogéneamente pobre, dado que cada barrio del Uruguay urbano cuenta con su propia escuela pública. En el ciclo de educación secundaria, sin embargo, dado que existen menos establecimientos, estos no suelen estar ubicados en barrios de contextos desfavorables.

Esto incide en que el peso de la heterogeneidad no se reparte homogéneamente por contexto social, sino que los estudiantes de mejores contextos no contribuyen a enriquecer el "efecto pares", mientras que los planteles educacionales ubicados en contextos más desfavorables tienden a recibir un alumnado heterogéneo y de un radio escolar mayor, dado que deben acoger a aquellos alumnos de contextos desfavorables de barrios que no cuentan con establecimientos educativos suficientes o que directamente no los tienen 5 .

Este proceso de segmentación por "descreme" (Filgueira y Bogliaccini, 2004) contribuye a la inequidad del sistema y es comparable con el proceso de segregación residencial por la vía de la generación de barrios privados en los sectores sociales de mayores recursos, que se ha producido en forma muy acelerada en la región en los últimos 30 años y en menor medida, pero acompañando la tendencia, en el Uruguay (Álvarez, 2007). Entre los estudios en que se ha examinado el efecto del establecimiento educativo en el rendimiento escolar destacan los realizados por Formichella (2011)

\footnotetext{
5 A diferencia del sistema de educación primaria, donde durante los últimos 20 años ha habido una pronunciada política de construcción de establecimientos educativos en los contextos más críticos, la expansión de los planteles de educación secundaria ha sido mucho más lenta.
}

sobre la Argentina; Oreiro y Valenzuela (2012) sobre el Uruguay, y Fernández y Del Valle (2013) sobre Costa Rica.

c) Provisión de materiales educativos y tecnológicos Los establecimientos educacionales carecen de recursos propios para mobiliarios y útiles escolares, a excepción de aquellos provistos por las comisiones de padres de alumnos. Por lo tanto, deben solicitar a la administración central los artículos necesarios. De lo contrario, debe buscarse dinero particular proveniente de la comisión de padres del establecimiento o de otros benefactores ocasionales (Da Silveira y Queirolo, 1998; Filgueira y Martínez, 2001).

Esto redunda en que existan diferencias importantes en materia de equipamiento didáctico y tecnológico debido a dos factores: la capacidad de negociación de los planteles educativos con el nivel central, y la capacidad económica de los padres de los alumnos. En un índice de equipamiento didáctico básico construido por los sistemas técnicos de la ANEP (1999), se señalaba que el $24 \%$ de los establecimientos educacionales de contexto muy desfavorable presentan carencias y que tan solo un $2 \%$ de aquellos de contexto favorable están en similar situación.

En resumen, en este artículo se sostiene que existen tres factores importantes de la regulación institucional del sistema educativo que atentan contra el abatimiento de la desigualdad de rendimiento: la descentralización a nivel docente para la distribución de los profesores por establecimiento educativo, el criterio de radio escolar para la distribución de alumnos por plantel de enseñanza, y la centralización a nivel nacional de la provisión de recursos educacionales y tecnológicos.

\section{III}

\section{Variables e hipótesis}

Para poner a prueba las tres hipótesis sugeridas, se propone un modelo logit con datos del Programa Internacional de Evaluación de Estudiantes (PISA por sus siglas en inglés) de 2006 para jóvenes de 15 años de edad, que se encuentran escolarizados en el ciclo básico de educación secundaria. En el cuadro 2 se resumen las variables integradas en el modelo, así como las hipótesis sugeridas.

PISA es una evaluación internacional de aprendizajes que se realiza sobre la base de una muestra representativa de alumnos de 15 años de edad de cada país, que asistan a la enseñanza secundaria. La evaluación se aplica cada tres años, y en cada uno de los ciclos de aplicación se evalúa un área cognitiva (Ciencias, Lectura o Matemática) con mayor profundidad. Además de la evaluación, en la prueba PISA se aplican tres cuestionarios para que sean contestados por los alumnos, sus familias y los directores de los establecimientos educativos. En 2006, el Uruguay también participó en la opción internacional de realizar una muestra dirigida a los alumnos del $2^{\circ}$ año del ciclo básico de secundaria, sin importar la 
edad. En la comparación entre los resultados de 2003 y 2006 se observan algunos cambios importantes: i) la cobertura de jóvenes de 15 años de edad se acrecienta del $75 \%$ al $80 \%$; ii) aumenta el porcentaje de alumnos que se encuentran rezagados, sobre todo en el primer año de secundaria; iii) se incrementa en cuatro puntos porcentuales la participación de los establecimientos de educación técnica, y iv) se duplica el porcentaje de alumnos que asisten a planteles de enseñanza con entorno muy desfavorable. En cuanto a los resultados, "entre 2003 y 2006 Uruguay tuvo una tendencia mixta: mantuvo su desempeño en Matemática pero retrocedió en Lectura" (ANEP, 2007, pág. 67).

La variable dependiente es "alfabetismo científico", una variable binaria construida a partir de la escala de competencias en la prueba PISA en Ciencias ${ }^{6}$. La encuesta PISA agrupa los puntajes de las pruebas por niveles que especifican las habilidades que el estudiante demuestra poseer. Los alumnos en el nivel 6 han logrado superar el máximo nivel de dificultad, y aquellos en el nivel 0 no han logrado realizar las tareas más básicas propuestas (véase el cuadro 1$)^{7}$. Se considera que los estudiantes en los niveles 0 y 1 no logran llegar al alfabetismo científico. En el reporte técnico de PISA se establece que "en el nivel 2 , los estudiantes posen un conocimiento científico adecuado para ofrecer posibles explicaciones a problemas en contextos familiares o basados en investigaciones simples. Son capaces de tener razonamiento directo y hacer interpretaciones literales de los resultados de investigaciones científicas o resolución de problemas tecnológicos" (OCDE, 2006b, pág. 294). En el año 2006, a modo de referencia, el $80,8 \%$ de los estudiantes que rindieron la prueba PISA en los países de la Organización para la Cooperación y el Desarrollo Económicos (OCDE) lograron ubicarse en el nivel 2 o superiores.

La elección de la variable dependiente responde a tres razones. En primer lugar, porque tiene una interpretación en términos sustantivos. Tal como se establece en el marco conceptual de la prueba PISA, las capacidades que definen el umbral de competencia científica —al igual que en las áreas de Lectura y Matemática-constituyen requisitos mínimos para una inserción razonable en la sociedad del conocimiento, en los estudios de nivel superior y en el mercado de trabajo.

\footnotetext{
6 En el programa PISA se hace rotar el énfasis de las pruebas entre Lengua, Matemática y Ciencias. En 2006, el énfasis fue en Ciencias. El mismo análisis podría repetirse con cualquiera de las otras dos áreas al tomar la base de datos de otros años (2003, 2009 o 2012).

7 Para una ampliación metodológica sobre cómo se construye esta variable, véase OCDE (2009, pág. 134).
}

CUADRO 1

\section{Escala de competencias en Ciencias}

\begin{tabular}{ccc}
\hline Niveles & $\begin{array}{c}\text { Porcentaje } \\
\text { de alumnos }\end{array}$ & Alfabetismo científico \\
\hline 0 & 15,3 & Por debajo de la línea \\
1 & 25,1 & $(40,4 \%)$ \\
2 & 32,1 & \\
3 & 18,5 & Por sobre la línea \\
4 & 7,4 & $(59,6 \%)$ \\
5 & 1,5 & \\
6 & 0,1 &
\end{tabular}

Fuente: elaboración propia sobre la base de datos de la Organización para la Cooperación y el Desarrollo Económicos (OCDE), PISA 2006 Technical Report, París, OECD Publishing, 2006.

En segundo lugar, en el caso uruguayo se ha constatado que, al controlar por el origen sociofamiliar, los estudiantes que a los 15 años no han desarrollado estas competencias tienen prácticamente vedada la finalización de la educación secundaria y, en consecuencia, el posterior tránsito a la educación superior (Bucheli y Casacuberta, 2000; Kaztman y Retamoso, 2007; Fernández, 2009; Fernández y otros, 2010). Asimismo, sus expectativas de inserción laboral se reducen casi completamente a empleos manuales, desprotegidos y con salarios comparativamente menores (Fernández y otros, 2010). Esto a su vez tiene importantes consecuencias en relación con los modelos de desarrollo a los que el país puede aspirar según los niveles de especialización del capital humano disponible.

El tercer argumento se refiere al problema de la equidad. La prueba PISA se compone de un conjunto de actividades escalables, lo que permite captar una amplia gama de habilidades jerárquicamente ordenables desde las más simples hasta las más complejas, que se expresan finalmente en una escala de valores continuos. Intuitivamente, un sistema que produce menor desigualdad podría pensarse como aquel en que los resultados de los alumnos tienen menor dispersión, lo que puede captarse fácilmente mediante indicadores clásicos como la varianza, el coeficiente de variación, el rango entre percentiles, o medidas como el coeficiente de Gini. Aunque estas medidas son estadísticamente potentes, es también razonable pensar el problema alternativamente desde el punto de vista de la capacidad del sistema educativo para garantizar el acceso de todos sus estudiantes al conjunto de aprendizajes establecidos según norma como necesarios para toda la población.

En los términos del primer abordaje, algunas situaciones de alta equidad pueden ser normativamente inaceptables - un sistema en el que todos sus estudiantes 
se ubicaran por debajo del umbral de competencias-. El segundo abordaje, al que se adhiere en este artículo, está menos preocupado por el hecho de que una élite académica se despegue de la media nacional y, en cambio, se concentra en la noción de universalización del acceso al núcleo básico de saberes que los sistemas educativos deberían garantizar. Estos abordajes no constituyen posiciones enfrentadas, sino complementarias, puesto que la evidencia empírica internacional señala que los sistemas con más altos desempeños son también aquellos que logran minimizar las brechas entre sus estudiantes (OCDE, 2008).

La variable incluida en el modelo para medir el efecto en el alfabetismo del primer factor de regulación del sistema considerado es la "proporción de docentes titulados en el establecimiento", medida como porcentaje de docentes con título sobre el total de profesores del plantel educativo (OCDE, 2006b, pág. 308). Dado que todos los establecimientos públicos del país debieran poseer un $100 \%$ de docentes titulados o distribuir en forma igualitaria entre establecimientos a los docentes con título del sistema, esta variable permite poner a prueba la hipótesis sobre el efecto negativo en la igualdad de aprendizaje que tiene el sistema de asignación docente a los planteles de enseñanza.

Hipótesis 1: el mecanismo de asignación docente hace que en los establecimientos educativos menos deseados se deban llenar los cargos con profesores no titulados, o bien en su último año de formación profesional o directamente ajenos a dicha formación.

$\mathrm{El}$ segundo factor considerado es "la heterogeneidad del establecimiento", medida como el desvío estándar respecto de la media de contexto socioeconómico de alumnos del plantel educativo.

Hipótesis 2: dada la distribución territorial de los establecimientos de educación secundaria, aquellos con mayor heterogeneidad tendrán una más elevada proporción de estudiantes por debajo del nivel de "alfabetismo".

El tercer factor considerado es la asimetría en la distribución de recursos tecnológicos y didácticos en el establecimiento educativo, como consecuencia de la centralización a nivel nacional del sistema de provisión y reparación de dichos recursos. Para captar este factor se utilizan dos variables. La "escasez de recursos tecnológicos" es un índice sumatorio simple de la escasez declarada por el director del establecimiento escolar respecto de computadoras disponibles para el aprendizaje, software para dichas computadoras y materiales audiovisuales. La segunda variable es la "escasez de recursos didácticos", medida como un índice sumatorio simple de la escasez declarada por el director del plantel educacional respecto de libros de texto y materiales de biblioteca ${ }^{8}$.

Hipótesis 3: a mayor escasez de recursos, en ambos casos, un menor porcentaje de alumnos en el establecimiento educativo alcanzan niveles de alfabetismo.

En el cuadro 2 se resumen los estadísticos descriptivos para las variables incluidas en el análisis (en el cuadro A.1 se detallan los estadísticos para cada grupo de la variable dependiente). Las variables incluidas como control a nivel de alumno son las siguientes: "sexo", "nivel socioeconómico del estudiante" y "condición de rezago" en su nivel de escolaridad. Estudios previos en el Uruguay coinciden en que en términos de "género", en promedio, las mujeres obtienen mejores resultados que los varones (Perera, Llambí y Messina, 2009) ${ }^{9}$. Dado que la variable dependiente en el presente modelo es "alfabetismo", se asume que en promedio la proporción de suficiencia por sobre el nivel de alfabetismo será mayor en las mujeres, pero no se tienen elementos para elaborar una hipótesis sobre la significación estadística de dicha diferencia de medias.

En relación con el "nivel socioeconómico del estudiante", se estima - en consonancia con la literatura sobre el tema- que la proporción de alfabetismo será mayor en los estudiantes de niveles socioeconómicos más altos.

Respecto de la "condición de rezago", la hipótesis planteada es que esta se relaciona en forma negativa con la proporción de alfabetismo. En este caso debe considerarse que la prueba PISA se realiza en estudiantes de 15 años de edad, por lo que no es posible comparar estudiantes con y sin rezago en el mismo nivel de educación secundaria (alumnos rezagados cursan niveles escolares inferiores al que les correspondería por edad).

Se incluyen, asimismo, dos variables de control a nivel de centro: "contexto socioeconómico del establecimiento educativo" y "tipo de establecimiento", según su carácter público o privado ${ }^{10}$. En el primer caso, se trabajó sobre

\footnotetext{
${ }^{8}$ La base de datos PISA ofrece un índice de recursos educativos de la escuela (SCMATEDU), que se calcula a partir de siete ítems con que se miden las percepciones del director del establecimiento sobre los posibles factores que dificultan la enseñanza en este. En el presente estudio no se utiliza dicho índice, porque se estima que es importante diferenciar conceptualmente la escasez de recursos tecnológicos de la escasez de recursos didácticos.

${ }^{9}$ En general, con respecto a los países participantes en el programa PISA, las mujeres obtienen mejor puntaje que los hombres en Lectura, pero los hombres lo obtienen en Ciencias y Matemática. Sin embargo, la edición 2006 de la prueba PISA para el Uruguay no arroja diferencias significativas, como se verá más adelante en los resultados del modelo estadístico utilizado.

${ }^{10}$ Son establecimientos urbanos (el Uruguay prácticamente no cuenta con planteles rurales), en tanto que las escuelas técnicas quedaron fuera del análisis.
} 
CUADRO 2

Características de las variables independientes

\begin{tabular}{|c|c|c|c|c|c|c|c|}
\hline \multirow{2}{*}{ Variable } & \multicolumn{2}{|c|}{ Operacionalización } & \multirow{2}{*}{$\begin{array}{l}\text { Efecto en } \\
\text { alfabetismo }\end{array}$} & \multirow{2}{*}{ Media } & \multirow{2}{*}{$\begin{array}{l}\text { Desvío } \\
\text { estándar }\end{array}$} & \multirow{2}{*}{$\begin{array}{l}\text { Valor } \\
\text { mínimo }\end{array}$} & \multirow{2}{*}{$\begin{array}{l}\text { Valor } \\
\text { máximo }\end{array}$} \\
\hline & $\begin{array}{l}\text { Nivel de } \\
\text { medición }\end{array}$ & Categorías & & & & & \\
\hline Sexo & $\begin{array}{l}\text { Variable } \\
\text { dummy }\end{array}$ & $0=$ mujer, 1 = varón & Negativo & 0,47 & 0,50 & 0 & 1 \\
\hline Contexto del alumno & Intervalo & Continua, positiva & Positivo & 0,54 & 0,17 & 0 & 1 \\
\hline $\begin{array}{l}\text { Proporción de docentes } \\
\text { titulados en el establecimiento } \\
\text { educativo }\end{array}$ & Intervalo & Continua & Positivo & 61,91 & 18,61 & 20 & 100 \\
\hline $\begin{array}{l}\text { Heterogeneidad del } \\
\text { establecimiento educativo }\end{array}$ & Intervalo & Continua & Positivo & 0,92 & 0,20 & 0,9 & 2,3 \\
\hline $\begin{array}{l}\text { Contexto del establecimiento } \\
\text { educativo }\end{array}$ & $\begin{array}{l}\text { Variable } \\
\text { ordinal }\end{array}$ & $\begin{array}{l}1=\text { muy desfavorable } \\
5=\text { muy favorable }\end{array}$ & Positivo & 3,00 & 1,23 & 1 & 5 \\
\hline \multirow[t]{2}{*}{ Escasez de textos y biblioteca } & Ordinal & $0=$ nula escasez & Negativo & 2,00 & 1,24 & 0 & 4 \\
\hline & & 4 = máxima escasez & & & 0,41 & & \\
\hline $\begin{array}{l}\text { Tipo de establecimiento } \\
\text { educativo }\end{array}$ & Nominal & $0=$ privado, $1=$ público & Negativo & 0,84 & 0,49 & 0 & 1 \\
\hline
\end{tabular}

Fuente: elaboración propia sobre la base de datos de Organización para la Cooperación y el Desarrollo Económicos (ocDE), PISA 2006 Technical Report, París, OEcD Publishing, 2006.

la base de una categorización realizada por la ANEP en el año 2006, donde los planteles escolares se agrupan en cinco categorías que van desde un entorno "muy favorable" hasta uno "muy desfavorable" (ANEP, 2007). Esta caracterización ha sido utilizada para comprobar la importancia que tiene el entorno del plantel de enseñanza en la obtención de resultados educativos de los estudiantes, donde queda claro que con un mejor entorno los alumnos muestran rendimientos superiores (ANEP, 2007). Como en el caso del contexto individual, la hipótesis es que la relación entre esta variable y la proporción de alfabetismo en el establecimiento educativo es positiva.
Finalmente, con respecto al "tipo de administración del establecimiento" y tomando como referencia el trabajo de Oreiro y Valenzuela (2012), la hipótesis es que los establecimientos educativos privados tendrán menor proporción de estudiantes alfabetos que los públicos. Es importante aclarar que en el contexto socioeconómico "muy desfavorable" no existe oferta privada, y en el contexto "muy favorable" no existe oferta pública. Por esto, el efecto de "tipo de establecimiento" en el alfabetismo se refiere a la comparación en los contextos "desfavorable", "medio" y "favorable", donde coexisten planteles educativos públicos y privados.

\section{IV}

\section{Técnica de análisis y resultados}

El modelo propuesto para el análisis es un logit binomial. La interpretación de los resultados se basa en el análisis de la probabilidad de cambio en la variable dependiente (entre 0 = analfabeto y 1 = alfabeto) dado un cambio de una unidad en la variable independiente, dejando el resto de las variables constantes en sus valores medios: $\operatorname{Pr}(Y=1 \mid x)$ (Gelman y Hill, 2007; Gujarati, 2004). El modelo incluye errores estándar robustos para corregir por heterocedasticidad ${ }^{11}$. El número de observaciones en el modelo es de 4.276, tomadas de la medición de 2006 de la prueba PISA para el caso del Uruguay. La

\footnotetext{
${ }^{11}$ Las pruebas para descartar multicolinealidad (factor de inflación de la varianza) y de homocedasticidad de los errores (prueba de BreuschPagan/Cook-Weisberg) se encuentran en los cuadros A.3 y A.4.
} 
tasa de participación bruta en la prueba fue del 98,2\% de los establecimientos educativos de la muestra (PISA requiere que participe el $85 \%$ de dichos planteles). La tasa de participación de los estudiantes que realizaron la prueba y contestaron el cuestionario alcanzó al 83,16\% de la muestra efectiva (PISA requiere que sea al menos del $80 \%$ ). En el cuadro 3 se presentan los resultados del modelo.

CUADRO 3

Efecto logit de variables seleccionadas en analfabetismo

$\begin{array}{ll}\text { Iteración 1: } \log \text { pseudolikelihood }= & -2878,3 \\ \text { Iteración } 4: \log \text { pseudolikelihood }= & -2250,6\end{array}$

Iteración 4: $\log$ pseudolikelihood $=$

\begin{tabular}{|c|c|c|c|c|c|}
\hline & $\beta$ & $\beta / 4$ & $\sigma^{2}$ Robusto & $\mathrm{Z}$ & Significancia \\
\hline Sexo & $-0,048$ & $-0,012$ & 0,090 & $-0,53$ & \\
\hline Contexto del alumno & 2,579 & 0,645 & 0,327 & 7,89 & $* * *$ \\
\hline Rezago del alumno & $-2,155$ & $-0,539$ & 0,156 & $-13,82$ & $* * *$ \\
\hline Proporción docentes titulados en establecimiento educativo & 0,009 & 0,002 & 0,003 & 3,49 & $* * *$ \\
\hline Heterogeneidad del establecimiento educativo & $-1,168$ & $-0,292$ & 0,297 & $-3,93$ & $* * *$ \\
\hline Contexto del establecimiento educativo & 0,459 & 0,115 & 0,059 & 7,82 & $* * *$ \\
\hline Escasez de materiales tecnológicos & $-0,069$ & $-0,017$ & 0,036 & $-1,89$ & $*$ \\
\hline Escasez de textos y biblioteca & 0,061 & 0,015 & 0,048 & 1,27 & \\
\hline Tipo de establecimiento educativo & 0,514 & 0,129 & 0,181 & 2,84 & $* *$ \\
\hline Intercepto & $-1,524$ & $-0,381$ & 0,427 & $-3,56$ & $* * *$ \\
\hline
\end{tabular}

$\mathrm{N}=4276 ;$ Wald chi2(9)=640; $\mathrm{LR}=1255 ; \mathrm{AIC}=4521$

Código: * $\mathrm{p}(\mathrm{Z})<0,05 ; * *<0,1 ; * * *<0,001$ (una cola)

Fuente: elaboración propia.

\section{Análisis de variables medidas a nivel de establecimiento educativo}

El modelo necesitó cuatro iteraciones para converger. Un primer análisis en el centro de los datos, dado que la pendiente de la curva logística es la más pronunciada en este punto, permite observar la magnitud máxima del efecto de cada variable independiente en el alfabetismo. Entre las variables a nivel de establecimiento, las más importantes en relación con los aspectos de la desigualdad educativa vinculados a la regulación del sistema —proporción de docentes titulados en el plantel educativo, homogeneidad de este, contexto del plantel, escasez de materiales tecnológicos y tipo de establecimiento- son estadísticamente significativas.

La proporción de docentes titulados en el establecimiento educativo es estadísticamente significativa para explicar el analfabetismo. Un aumento del 10\% en dicha proporción en el centro de los datos y manteniendo las otras variables constantes en sus promedios, incrementa en $2 \%$ la probabilidad de obtener resultados por nivel de alfabetismo.

Este resultado apoya la hipótesis planteada sobre el carácter negativo de la regulación para la elección de horas docentes en el sector público, en la que opera únicamente un criterio estratégico del propio docente. Este profesor solo está limitado por las estrategias de aquellos colegas que le precedan en el proceso de elección. Tal mecanismo opera a un nivel máximo de descentralización (considerando nivel de la administración central de la educación pública, nivel de establecimiento y nivel docente) que no permite incluir correctivos en el sistema para mejorar la equidad en la distribución de recursos entre establecimientos de enseñanza.

No obstante, el efecto es pequeño aunque significativo, lo que es esperable dado que la variable utilizada como variable proxy captura la diferencia en niveles de titulación (y presumiblemente experiencia docente), pero no así el efecto de la rotación de profesores ${ }^{12}$. De todos modos, el análisis del modelo presenta evidencia suficiente para señalar decididamente a este problema como causa probable y relevante de la desigualdad educativa.

La heterogeneidad del establecimiento es también estadísticamente significativa y se vincula en forma negativa con la probabilidad de salir del analfabetismo. Cuanto menor es la heterogeneidad socioeconómica del alumnado del plantel, mayor es la probabilidad del alumno de obtener resultados por sobre la línea de alfabetismo. En el centro de los datos, la heterogeneidad del establecimiento educativo en el Uruguay es de un (1) desvío estándar respecto de la media del contexto del establecimiento. En este caso, el aumento de la heterogeneidad de dicho

\footnotetext{
12 El programa PISA no provee un indicador mejor y la ANEP no ha hecho públicas mediciones sistemáticas de este fenómeno.
} 
plantel en una categoría (de 0 a 1), acrecienta en $29 \%$ la probabilidad de salir del analfabetismo.

Los resultados sustentan la hipótesis planteada sobre el efecto regresivo que la regulación de asignación de estudiantes a establecimientos educativos — por radio escolar - tiene en la probabilidad de que un alumno salga del analfabetismo. En un contexto de media y alta segregación residencial, el sistema de radio escolar segmenta al alumnado impidiendo una distribución de este que favorezca un "efecto pares" positivo.

La variable incluida en el modelo para medir escasez de libros de texto y materiales de biblioteca no resulta estadísticamente significativa para explicar el nivel de alfabetismo. Sin embargo, la variable sobre escasez de materiales tecnológicos sí lo es. En el centro de los datos, un aumento en una categoría en términos de escasez de materiales tecnológicos reduce en $1,7 \%$ la probabilidad del estudiante de obtener resultados que superen el nivel de analfabetismo ${ }^{13}$.

En definitiva, la hipótesis sobre el efecto inequitativo que tiene la centralización de los mecanismos de provisión de materiales educativos es corroborada por el modelo presentado en este trabajo solo para el caso de los materiales tecnológicos, lo que resulta de extrema importancia para comprender los riesgos vinculados a la equidad e integración del individuo en sociedad que encierra la brecha tecnológica.

\section{Análisis de tres escenarios típicos}

A objeto de ejemplificar los efectos de las variables introducidas en el modelo, se presenta una serie de escenarios donde, a partir de una situación tipo, se observan las probabilidades de que el estudiante obtenga un resultado superior a la línea de alfabetismo científico (niveles 2 a 6). Para mayor claridad respecto de las hipótesis que orientan el estudio, los escenarios se conforman dejando constantes las características del alumno (el alumno promedio del sistema) y haciendo variar las características del establecimiento de enseñanza, de modo de poder observar cómo cambia la probabilidad de este alumno promedio de obtener puntajes sobre el nivel de alfabetismo, dependiendo de si estudia en un plantel educativo promedio, en uno que se encuentra en el $25 \%$ con las peores características, o en otro en el $25 \%$ con las mejores características.

\footnotetext{
13 La prueba PISA de 2006 se realizó antes de que el Gobierno del Presidente Vázquez iniciara el Plan CEIBAL, mediante el cual se dota progresivamente de un computador personal a cada alumno en establecimientos de educación primaria del sector público.
}

\section{a) Escenario 1}

Para el año 2006, el individuo promedio de la muestra es una alumna de contexto socioeconómico medio (categoría 3), que no sufrió años de rezago en el sistema y que estudia en un establecimiento educativo promedio con las siguientes características: es un plantel público, el promedio de alumnos que asisten es también de contexto sociocultural medio, la heterogeneidad del establecimiento también se encuentra en el promedio de la distribución ( 0,9 desvíos estándar), el $62 \%$ de los docentes tienen título, al menos dos de las tres categorías incluidas en el índice de carencias tecnológicas presentan dichas carencias, y existe insuficiencia en al menos una categoría de materiales didácticos.

La probabilidad de que esta alumna obtenga resultados en la prueba PISA que superen el nivel de analfabetismo es del 74\%; es decir, que 3 de cada 4 de estas alumnas hipotéticas obtendrán un puntaje en la prueba que les permitirá sobrepasar el nivel 1 (véase el cuadro 1). Si la alumna tuviera algún año de rezago, su probabilidad de superar el nivel de analfabetismo sería de solo un $25 \%$. Este dato es sumamente importante, dado que el Uruguay se ubica entre los 4 países con mayores niveles de rezago a los 15 años de edad ${ }^{14}$ entre todos los países del mundo donde se realiza la prueba PISA.

\section{b) Escenario 2}

Se ubica a la misma alumna en un plantel educativo cuyas características lo posicionen entre el $25 \%$ de los mejores del país. En este establecimiento, el promedio de alumnos que asiste es de contexto sociocultural medio (es decir, que el efecto pares queda constante en esta comparación), la heterogeneidad es menor ( 0,72 desvíos estándar), el $73 \%$ de los profesores tienen título docente, y solo existen carencias tecnológicas en dos de las tres categorías, y didácticas en una sola. La probabilidad de esta misma alumna de obtener resultados que superen el nivel de analfabetismo es ahora del $82 \%$ (respecto del $74 \%$ del escenario 1). Si la alumna ha sufrido al menos un año de rezago, dicha probabilidad baja a un $34 \%$.

\section{c) Escenario 3}

Finalmente, supóngase a la misma alumna en un establecimiento educativo cuyas características lo posicionen entre el $25 \%$ de los peores planteles del país. En este, el promedio de alumnos que asisten es de contexto

\footnotetext{
14 El Uruguay tiene porcentajes muy altos de abandono del sistema a los 15 años y rezago (14\% de quienes logran permanecer a la edad de 15 años). Esto representa una solución ineficiente al problema generado por la tensión inherente a todo sistema entre cobertura y calidad.
} 
sociocultural desfavorable, su heterogeneidad es mayor (1,05 desvíos estándar), solo el 47\% de los profesores tienen título docente, y existen carencias tecnológicas en las tres categorías y didácticas en las dos categorías. La probabilidad de que esta misma alumna obtenga

\section{V}

\section{Conclusiones}

El análisis aquí presentado evidencia un escenario negativo con respecto al efecto de los mecanismos de asignación docente, asignación de estudiantes a establecimientos y distribución de materiales didácticos en la producción y reproducción de las desigualdades educativas. En primer lugar, el mecanismo de asignación de docentes a los establecimientos educativos produce una segmentación de la oferta de acuerdo con su contexto sociocultural, lo que refuerza las desigualdades de origen de los alumnos. En segundo lugar, el mecanismo de asignación de estudiantes a los planteles de enseñanza produce homogeneidad en los establecimientos educacionales de alumnos de contextos favorables, y heterogeneidad del alumnado en los contextos medios, desfavorables y muy desfavorables. Finalmente, el mecanismo de provisión y mantenimiento de recursos tecnológicos al servicio de la educación refuerza un acceso desigual a estos en favor de los establecimientos educativos de contextos más favorables.

El efecto que los mecanismos de asignación de cargos docentes tienen en la distribución de capacidades entre los establecimientos educativos y la consecuente generación de inequidades en calidad y equidad plantean el problema de renunciar a operar según la "lógica de mercado" que dicho mecanismo impone. Este es un claro problema de "principal agente", en el que la divergencia entre los intereses del sistema y los del cuerpo docente es notoria, y cabe preguntarse cuál es el objetivo que persigue el sistema al permitir a los docentes elegir establecimiento sin poder influir en el mecanismo.

La asignación de estudiantes a los planteles de enseñanza según radio escolar reproduce en su interior patrones de segregación residencial, con la consecuente producción de segmentación educativa. En la reforma educacional de 1995 se discutieron algunos proyectos tendientes a romper esta dinámica, tales como una política de buses escolares que permitieran trasladar estudiantes entre zonas de la ciudad, pero esta no prosperó por resultados que superen el nivel de analfabetismo es de solo el $58 \%$ (comparado con el $74 \%$ en el escenario 1 y el $82 \%$ en el escenario 2). Si la alumna ha sufrido al menos un año de rezago, dicha probabilidad desciende a solo un $14 \%$. razones tanto presupuestarias como políticas. El riesgo de no implementar políticas orientadas a quebrar esta lógica es la profundización de dicha segmentación, en la medida que el sistema público se expanda mediante la construcción de planteles de enseñanza en barrios de contextos muy desfavorables, generando entonces un problema similar al que enfrenta actualmente la educación primaria: la producción de homogeneidad cultural en barrios pobres.

La fuerte centralización del sistema educativo nacional ha comenzado a evidenciar signos de inadecuación en diversos aspectos de su gestión. La brecha tecnológica será, sin lugar a dudas, un problema neurálgico en términos de la superación de la desigualdad de oportunidades en el tiempo venidero, y el sistema educacional uruguayo no parece estar preparado para abordarlo. No obstante, el Plan CEIBAL ha logrado reducir enormemente la brecha tecnológica a nivel de educación primaria y va en camino de hacer lo propio en educación secundaria. Sin embargo, esta solución exitosa no debiera hacer olvidar el problema de origen, que radica en el diferencial de acceso de los distintos establecimientos a los recursos y en el problema de refuerzo de las desigualdades en el origen que esto trae consigo. Asimismo, no debe pasar desapercibido que este plan fue diseñado desde fuera del sistema educativo nacional (operado por una agencia de innovación tecnológica), debido en parte a los conflictos internos y a la oposición a dicha política, incluso a fines de 2010, por parte del cuerpo docente del sistema.

En conclusión, los desafíos institucionales que enfrenta el sistema educativo nacional para superar los problemas de cobertura, calidad y equidad requieren acciones a dos niveles. En primer lugar, deben replantearse los objetivos del ciclo de educación secundaria y, consecuentemente, los mecanismos institucionales que se ponen al servicio de dichos objetivos. Este ciclo educativo no opera en la actualidad como un proceso de 
formación orientado al acceso a la universidad, sino que debe compartir este objetivo con la formación orientada a una inserción adecuada de los jóvenes en el mercado de trabajo y en carreras técnicas de nivel terciario no universitario. En segundo lugar, pero asociado a lo primero, el sistema educacional debe superar problemas de corporativismos sectoriales, de modo de poder avanzar hacia los cambios institucionales requeridos para privilegiar la educación de los alumnos por sobre los intereses de los actores del sistema.

Finalmente, el estudio aquí presentado tiene sus propias limitaciones, referidas al análisis de solo un sector de los aspectos institucionales que pueden afectar a la equidad en el logro educativo. Por una parte, en el Uruguay no se han incorporado factores tales como exámenes centralizados, rendición de cuentas, elección de establecimientos (school choice) e incentivos al desempeño. En consecuencia, no es posible valorar el eventual efecto de estos factores. Por otra parte, las limitaciones se refieren a la información disponible en las bases de datos del programa PISA y en cómo lograr acercarse con estos datos a la medición de los conceptos deseados. En tal sentido, es de esperar que futuros aportes puedan abordar con éxito estas limitaciones.

ANEXO

CUADRO A.

Estadísticos descriptivos de variables incluidas en el análisis, por categoría de variable dependiente

\begin{tabular}{|c|c|c|c|c|}
\hline \multirow{2}{*}{ Variable } & \multicolumn{2}{|c|}{ No alfabetizados } & \multicolumn{2}{|c|}{ Alfabetizados } \\
\hline & Media & Desvío estándar & Media & Desvío estándar \\
\hline Contexto del alumno & 0,47 & 0,16 & 0,59 & 0,16 \\
\hline Rezago del alumno & 0,33 & 0,47 & 0,03 & 0,17 \\
\hline Proporción de docentes titulados del establecimiento educativo & 56,77 & 18,13 & 63,10 & 18,49 \\
\hline Heterogeneidad del establecimiento & 0,96 & 0,18 & 0,89 & 0,21 \\
\hline Contexto del establecimiento & 2,00 & 1,00 & 3,02 & 1,19 \\
\hline Escasez de materiales tecnológicos & 3,79 & 1,73 & 2,89 & 2,00 \\
\hline Escasez de textos y biblioteca & 2,24 & 1,15 & 1,77 & 1,26 \\
\hline Tipo de establecimiento & 0,92 & 0,27 & 0,71 & 0,46 \\
\hline
\end{tabular}

Fuente: elaboración propia sobre la base de datos de la Organización para la Cooperación y el Desarrollo Económicos (OCDE), PISA 2006 Technical Report, París, OECD Publishing, 2006.

CUADRO A. 2

Descripción de variables utilizadas en el análisis

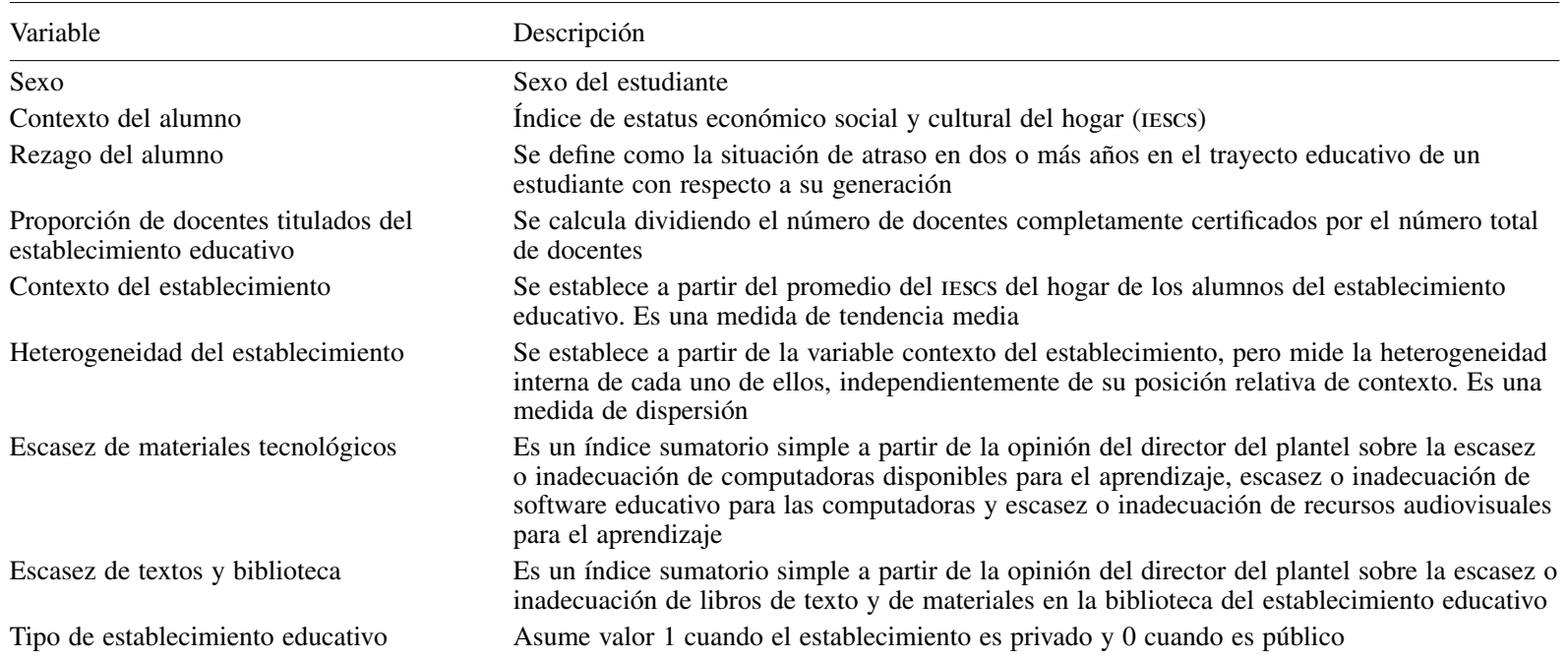

Fuente: elaboración propia. 
CUADRO A.3

Factor de inflación de varianza (VIF) para modelo

\begin{tabular}{lcc}
\hline Variable & VIF & $1 / \mathrm{VIF}$ \\
\hline Sexo & 2,58 & 0,387055 \\
Contexto del alumno & 2,47 & 0,405523 \\
Rezago del alumno & 2,24 & 0,446160 \\
Proporción de docentes titulados del establecimiento educativo & 2,03 & 0,492614 \\
Heterogeneidad del establecimiento & 1,56 & 0,639929 \\
Contexto del establecimiento & 1,24 & 0,804850 \\
Escasez de materiales tecnológicos & 1,17 & 0,851628 \\
Escasez de textos y biblioteca & 1,06 \\
Tipo de establecimiento educativo & 0,941546 \\
Media vIF & 1,02 & 0,982756 \\
\end{tabular}

Fuente: elaboración propia sobre la base de datos de Organización para la Cooperación y el Desarrollo Económicos (OCDE), PISA 2006 Technical Report, París, OECD Publishing, 2006.

CUADRO A.4

\section{Prueba de Breusch-Pagan/Cook-Weisberg para heterocedasticidad}

Ho: varianza constante

Variables: valores ajustados de analfabetismo

$\chi^{2}(1)=9,27$

$\mathrm{P}>\chi^{2}=0,0023$

Fuente: elaboración propia sobre la base de datos de Organización para la Cooperación y el Desarrollo Económicos (OCDE), PISA 2006 Technical Report, París, OECD Publishing, 2006.

\section{Bibliografía}

Abbott, W., P. Tyler y C. Wallace (1990), An Introduction to Sociology: Feminist Perspectives, Nueva York, Routledge.

Álvarez, M.J. (2007), "Golden ghettos: Gated communities and class residential segregation in Montevideo", Environment and Planning A, vol. $39, \mathrm{~N}^{\circ} 1$.

ANEP (Administración Nacional de Educación Pública) (2008), Censo Nacional Docente, Montevideo, División de Investigación, Evaluación y Estadística. (2007), Uruguay en PISA 2006, Montevideo.

(2005), Uruguay en el Programa PISA. Boletín Informativo, $\mathrm{N}^{\circ}$ 5, Montevideo [en línea] http://www.anep.edu.uy/ anep/phocadownload/pisa/pisa2003/Boletines/Boletin\%20 informativo\%2005\%20-\%202003.pdf.

(2002), "La repetición en la escuela pública en cifras", Serie Estadística, $\mathrm{N}^{\mathrm{o}} 3$, Montevideo, Gerencia General de Planeamiento y Gestión Educativa.

(1999), Estudio de los factores institucionales y pedagógicos que inciden en los aprendizajes en escuelas primarias de contextos sociales desfavorecidos en el Uruguay, Montevideo.

Banco Mundial (2014), "Gini Index Ranking" [en línea] http://data. worldbank.org/.

Bogliaccini, J. (2007), "Primary education: Changing Mainstay of Uruguay", International Handbook of Urban Education, G. Noblit y D. Pink (eds.), Springer.

(2003), "La autonomía del director de escuela", Revista Umbral 2000, $\mathrm{N}^{\circ} 11$
Bucheli, M. y C. Casacuberta (2000), “Asistencia escolar y participación en el mercado de trabajo de los adolescentes en Uruguay", El Trimestre Económico, vol. 67, N²67, Fondo de Cultura Económica.

Coleman, J. (1966), Equality of Educational Opportunity, Washington, D.C., Departamento de Educación.

Cueto, S. (2004), "Factores predictivos del rendimiento escolar, deserción e ingreso a educación secundaria en una muestra de estudiantes de zonas rurales del Perú".

Da Silveira, P. y R. Queirolo (1998), "Análisis organizacional: Cómo funciona la educación pública en Uruguay", Estudios, $\mathrm{N}^{\circ} 6$, Montevideo, Centro de Estudios de la Realidad Económica y Social (CERES).

De Giorgi, G., M. Pellizzari y S. Redaelli (2010), "Identification of social interactions through partially overlapping peer groups", American Economic Journal: Applied Economics, vol. 2, $\mathrm{N}^{\circ} 2$, Nashville, Tennessee, American Economic Association.

DiMaggio, P. (1982), "Cultural capital and school success: The impact of status culture participation on the grades of Us high school students", American Sociological Review, vol. 47, $\mathrm{N}^{\circ} 2$, American Sociological Association.

Edmonds, R. (1986), "Characteristics of effective schools", The School Achievement of Minority Children: New Perspectives, U. Neisser (ed.), Routledge.

Fernández, T. (2009), "La desafiliación en la educación media en Uruguay. Una aproximación con base en el panel de estudiantes 
evaluados por PISA 2003", Revista Iberoamericana sobre Calidad, Eficacia y Cambio en Educación (REICE), vol. 7, $\mathrm{N}^{\circ} 4$. (1999), "Análisis organizacional en educación", Documento de Trabajo, $\mathrm{N}^{\mathrm{o}} 46$, Montevideo, Facultad de Ciencias Sociales de la Universidad de la República.

Fernández, T. y otros (2010), La desafiliación en la Educación Media y Superior de Uruguay. Conceptos, estudios y políticas, Montevideo, Universidad de la República.

Fernández, A. y R. del Valle (2013), "Desigualdad educativa en Costa Rica: La brecha entre estudiantes de colegios públicos y privados. Análisis con los resultados de la evaluación internacional PISA", Revista de la CEPAL, $\mathrm{N}^{\circ} 111$ (LC/ G.2597-P), Santiago de Chile.

Filgueira, F. y E. Martínez (2001), La reforma educativa en Uruguay: Desafíos y tendencias, Proyecto "Alcance y resultados de las reformas educativas en Argentina, Chile y Uruguay", Buenos Aires, Banco Interamericano de Desarrollo (BID).

Filgueira, F. y J.A. Bogliaccini (2004), "Las reformas educativas en Chile y Uruguay: Descentralización orientada al mercado versus centralismo estatista", Descentralización y políticas sociales en América Latina, R. Gomà y J. Jordana (eds.), Barcelona, Centro de Información y Documentación Internacionales en Barcelona (CIDOB).

Filgueira, C., A. Fuentes y F. Rodríguez (2006), "Viejos instrumentos de la inequidad educativa: Repetición en primaria y su impacto sobre la equidad en Uruguay", Educación y brechas de equidad en América Latina, S. Cueto (ed.), Santiago de Chile, Fondo de Investigaciones Educativas.

Filgueira, F. y otros (2005), "Estructura de riesgo y arquitectura de protección social en el Uruguay actual: Crónica de un divorcio anunciado", Prisma, $\mathrm{N}^{\circ} 21$.

Formichella, M.M. (2011), “Se debe el mayor rendimiento de las escuelas de gestión privada en la Argentina al tipo de administración?", Revista de la CEPAL, N 107 (LC/G.2508-P), Santiago de Chile.

García Huidobro, J.E. (2003), "La buena escuela", La centralidad de la escuela en la política educativa chilena de los 90, J.E. García Huidobro y C. Sotomayor, Santiago de Chile. (2000), "La deserción y el fracaso escolar", Educación, pobreza y deserción escolar, Santiago de Chile, Fondo de las Naciones Unidas para la Infancia (UNICEF).

Gelman, A. y J. Hill (2007), Data Analysis Using Regression and Multilevel/Hierarchical odels, Cambridge, Cambridge University Press.

Graham, B.S. (2008), "Identifying social interactions through conditional variance restrictions", Econometrica, vol. 76, $\mathrm{N}^{\circ} 3$, Nueva York, The Econometric Society.

Gujarati, D. (2004), Basic Econometrics, McGraw-Hill.

Hanushek, E. y L. Woessmann (2010), "The economics of international differences in educational achievement", CESifo Working Papers Series, $\mathrm{N}^{\circ}$ 3037, Munich, cEsifo Group.

Hanushek, E., S. Link y L. Woessmann (2012), "Does school autonomy make sense everywhere? Panel estimates from Pisa", NBER Working Paper, $\mathrm{N}^{\circ}$ 17591, Cambridge, Massachusetts, National Bureau of Economic Research.

Hanushek, A. y J. Luque (2001), "Efficiency and equity in schools around the world", Economics of Education Review, vol. 22, $\mathrm{N}^{\circ} 5$, Amsterdam, Elsevier.

Kaztman, R. (2001), "Seducidos y abandonados: el aislamiento social de los pobres urbanos", Revista de la CEPAL, $\mathrm{N}^{\circ} 75$ (LC/G.2150-P), Santiago de Chile.
(1999), Activos y estructura de oportunidades: Estudios sobre las raíces de la vulnerabilidad social en Uruguay (LC/ MVD/R.180), Montevideo, Comisión Económica para América Latina y el Caribe (CEPAL)/Programa de las Naciones Unidas para el Desarrollo (PNUD).

Kaztman, R. y F. Filgueira (2001), Panorama de la infancia y la familia en Uruguay, Montevideo, Universidad Católica del Uruguay.

Kaztman, R. y A. Retamoso (2007), "Efectos de la segregación urbana sobre la educación", Revista de la CEPAL, $\mathrm{N}^{\circ} 91$ (LC/G.2333-P), Santiago de Chile.

(2006), Aprendiendo Juntos. Retos a la educación desde los procesos de segregación urbana, Montevideo, Universidad Católica del Uruguay.

Martínez, A.L. (coord.), S. Alonso y D. Díaz (2009), Monitoreo y evaluación de impacto social del Plan CEIBAL. Metodología y primeros resultados a nivel nacional, Montevideo [en línea] http://www.ceibal.org.uy.

Méndez, N. y M. Zerpa (2009), "Desigualdad en las capacidades educativas en Uruguay y Chile", documento en el I Seminario sobre Economía de la Educación, Montevideo, Facultad de Ciencias Económicas y Administración, Universidad de la República.

OCDE (Organización para la Cooperación y el Desarrollo Económicos) (2009), PISA Data Analysis Manual. Second Edition, París, OECD Publishing.

(2008), Informe PISA 2006. Competencias científicas para el mundo del mañana, Santillana.

(2006a), "Database-PISA 2006" [en línea] http://pisa2006. acer.edu.au/.

(2006b), PISA 2006 Technical Report, París, OECD Publishing.

Oreiro, C. y J.P. Valenzuela (2012), "Factores que determinan el desempeño educativo en el Uruguay 2003-2006", Revista de la CEPAL, $\mathrm{N}^{\circ} 107$ (LC/G.2536-P), Santiago de Chile.

Perera, M., C. Llambí y P. Messina (2009), "Desigualdad de oportunidades y el rol del sistema educativo en los logros de los jóvenes uruguayos", Documento de Trabajo, $\mathrm{N}^{\circ} 4$, Montevideo, Centro de Investigaciones Económicas (CINVE).

Rama, G. (1993), ¿Qué aprenden y quiénes aprenden en las escuelas públicas de Uruguay?, Montevideo, oficina de la CEPAL en Montevideo.

Reimers, F. (2000), Unequal Schools, Unequal Chances, Cambridge, Massachusetts, Harvard University Press.

Roemer, J. (2005), "Equality of opportunity", The New Palgrave Dictionary of Economics. S.N. Durlauf y L.E. Blume (eds.), Palgrave Macmillan.

Sacerdote, B. (2001), "Peer effects with random assignment: results for Dartmouth roommates", Quarterly Journal of Economics, vol. 116, $\mathrm{N}^{\circ} 2$, Oxford, Oxford University Press.

Stromquist, N.P. (1990), "Gender inequality in education: Accounting for women's subordination", British Journal of Sociology of Education, vol. 11, $\mathrm{N}^{\mathrm{o}}$ 2, Taylor \& Francis.

Vélez, E., E. Schiefelbein y J. Valenzuela (1994), "Factores que afectan el rendimiento académico en la educación primaria", Revista Latinoamericana de Innovaciones Educativas, $\mathrm{N}^{\circ} 17$, Buenos Aires, Organización de Estados Iberoamericanos para la Educación, la Ciencia y la Cultura (OEI).

Wilkinson, I. y otros (2002), "Discussion: Modeling and maximizing peer effects in school", International Journal of Educational Research, vol. $37, \mathrm{~N}^{\circ} 5$. 